\title{
Telomere-related Genome Instability in Cancer
}

\author{
T. DE LANGE \\ The Rockefeller University, New York, New York 10021
}

\begin{abstract}
Genome instability is a hallmark of most human cancers. Although a mutator phenotype is not required for tumorigenesis, it can foster mutations that promote tumor progression. Indeed, several inherited cancer-prone syndromes are due to mutations in DNA repair pathways. However, sporadic tumors are usually proficient in DNA repair, making it unlikely that unrepaired lesions are a major source of genome instability in sporadic cancers. A decade ago, I argued in another CSHL Press publication that a "collapse in telomere function can explain a significant portion of the genetic instability in tumors" (de Lange 1995). Since that time, the structure of mammalian telomeres has been analyzed, the consequences of telomere dysfunction have been determined, a mouse model for cancer-relevant aspects of telomere biology has been developed, and the nature and magnitude of cancer genome rearrangements have been revealed. In light of these developments, this is an opportune time to revisit the conjecture that telomere dysfunction contributes to genome instability in human cancer.
\end{abstract}

The genomes of human carcinomas and several other tumor types are in an astonishing state of disarray. The extent of genome scrambling was only appreciated after development of high-resolution techniques. Spectral karyotyping (M-FISH, SKY) has painted a picture of extensive reshuffling of chromosome segments. Techniques that display the differences between normal and cancer genomes, combined with DNA microarrays (array-CGH, Pinkel et al. 1998; ROMA, Lucito et al. 2003), have revealed countless copy number changes. What is the origin of this genome instability? Recent findings make a compelling case for the view that genome instability in human cancer is largely rooted in telomere dysfunction. Dysfunctional telomeres can explain most genome alterations observed in human cancer (Fig. 1). Moreover, the telomere- shortening process that gives rise to dysfunctional telomeres takes place in the majority of human somatic cells, potentially explaining genome instability in many different human tumor types. Finally, a brief period of telomere dysfunction early in tumorigenesis can explain the transient nature of cancer genome instability. New data relating to these issues are discussed below.

\section{TELOMERES AND THEIR FUNCTIONAL COLLAPSE}

The molecular features of human telomeres are now understood in sufficient depth to permit formulation of a working model for how these elements protect chromosome ends (Fig. 2). In human cells, chromosomes termi-
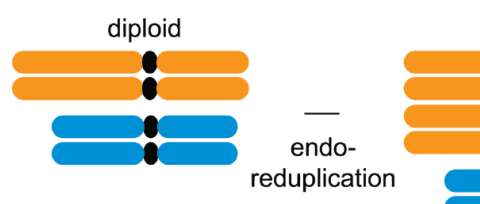

tetraploid
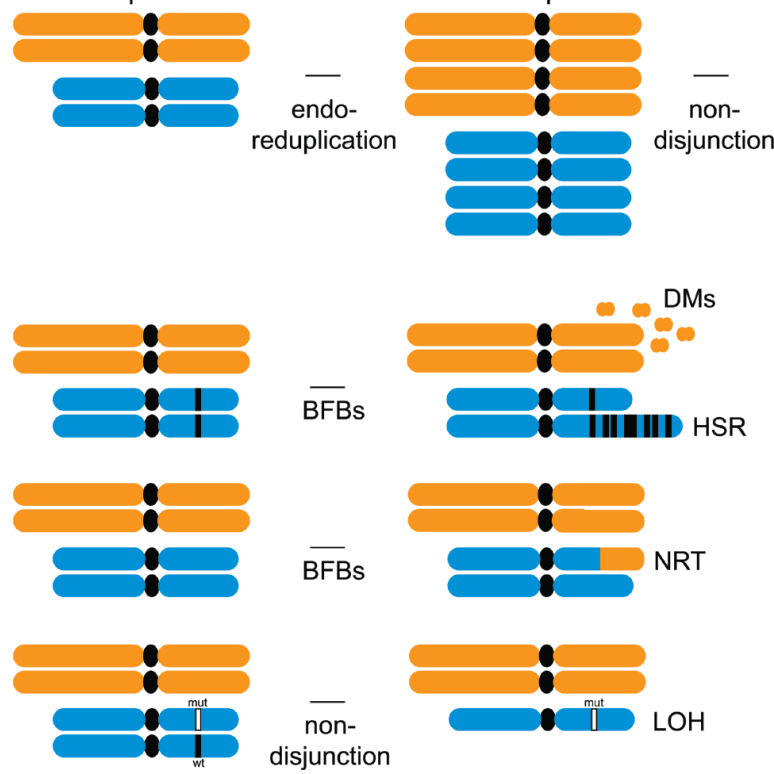

$\overline{\text { non- }}$

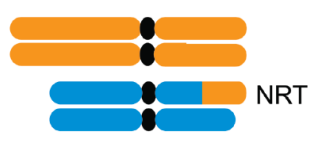

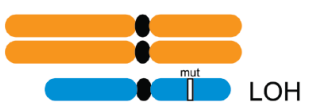

Figure 1. Telomere-related genome instability. Schematic of various types of karyotypic alterations that can be the result of telomere dysfunction. 


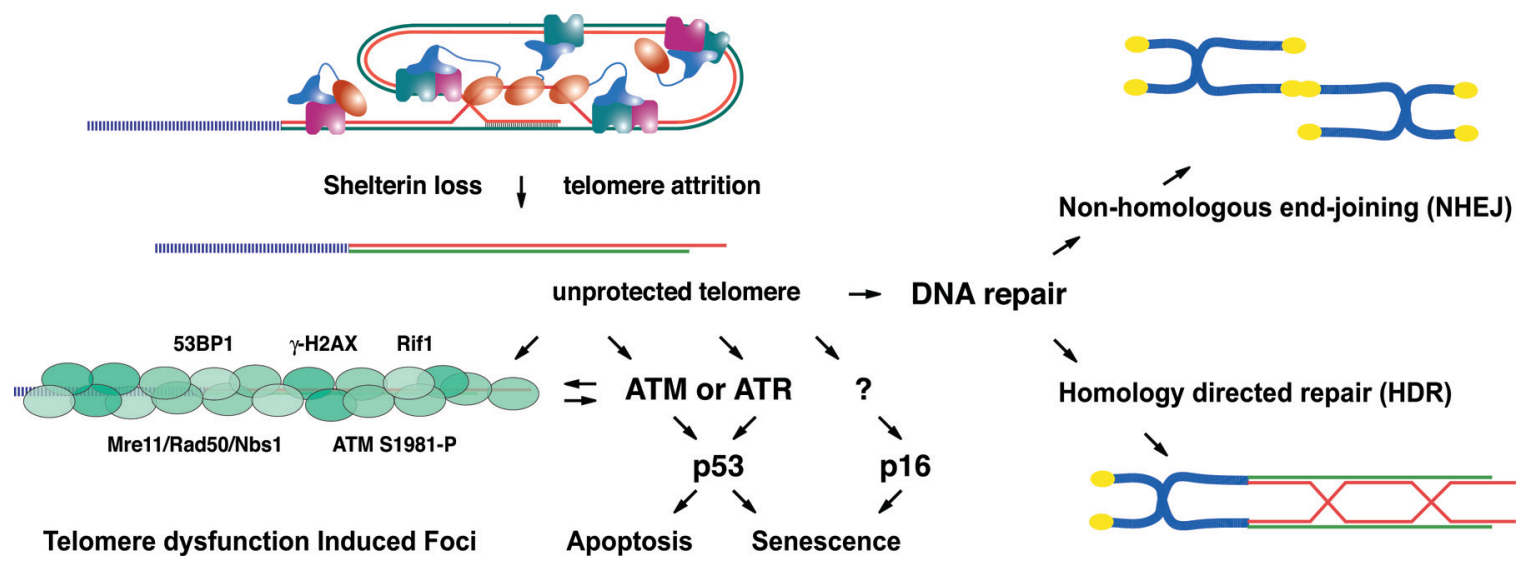

Figure 2. Telomere function and dysfunction. Schematic of the mammalian telomeric complex in the t-loop configuration associated with shelterin. The consequences of shelterin inhibition (through TRF2 deletion) and telomere attrition are depicted below.

nate in a long array of direct repeats that originate from telomerase, a telomere-specific reverse transcriptase with an RNA component that contains the template for the telomeric TTAGGG sequence (Chen and Greider 2005; Cristofari and Lingner 2005). Although DNA replication leads to progressive loss of telomeric DNA, most human and mouse cells have an adequate telomere reserve for extensive proliferation without telomerase. Ultimately, however, telomere attrition limits the proliferative life span of human cells so that activation of telomerase or an alternative pathway for telomere lengthening (ALT) is required for cellular immortalization (Neumann and Reddel 2005; Shay and Wright 2005). The ability of telomerase to counteract telomere attrition explains its virtual omnipresence in human cancer.

At the end of the telomeric repeat array, singlestranded TTAGGG repeats form a 50-300-nucleotide 3' overhang. This tail is thought to be important for telomere function. EM analysis showed that the singlestranded TTAGGG repeats pair with CCCTAA repeats within the duplex telomeric repeat array, generating a large double-stranded loop, the t-loop (de Lange 2005a). Because the t-loop conceals the physical end of the chromosome, it might explain how cells distinguish natural chromosome ends from double-strand breaks (DSBs), but other aspects of the telomeric complex are likely to contribute to the protective function of telomeres as well.

The telomeric DNA is associated with a telomere-specific protein complex, called shelterin (de Lange 2005b). Shelterin contains three telomeric DNA-binding proteins (TRF1, TRF2, and POT1) that together confer exquisite specificity for the sequence and structure of telomeric DNA. The complex is sufficiently abundant at chromosome ends to coat the whole duplex telomeric repeat array. Shelterin is at telomeres throughout the cell cycle and present in all human cells regardless of their proliferative state. Unlike telomerase, deletion of most shelterin components results in embryonic lethality in the mouse.

Shelterin has three main functions at telomeres. It protects telomeres from DNA repair enzymes and helps conceal chromosome ends from the DNA damage response signaling pathways. The third function of shelterin is to govern telomere length. Shelterin can control telomerase through a cis-acting negative feedback loop that maintains telomeres within a set size range (Smogorzewska and de Lange 2004). The challenge is to understand how shelterin and its associated factors execute these functions. In part, the answer may be found in the DNA remodeling activities of TRF1 and TRF2. Both proteins alter telomeric DNA into looped structures, suggesting that they promote t-loop formation in vivo. The t-loop structure has been invoked as an architectural mechanism to conceal chromosome ends from the repair enzymes that threaten telomere integrity and could explain why the DNA damage response does not get activated by natural chromosome ends.

Telomere function collapses when shelterin is inhibited or when the telomeric DNA has been shortened beyond a critical (but as yet undefined) minimal length. These two sources of telomere dysfunction have similar outcomes, suggesting that shortened telomeres fail to function because of insufficient loading of shelterin. Studies of the consequences of telomere attrition and shelterin inhibition have illuminated the types of genome damage resulting from telomere dysfunction.

\section{TELOMERE-RELATED GENOME INSTABILITY}

\section{The Root Cause: Repair of Dysfunctional Telomeres}

Telomere-related genome instability is caused by inappropriate DNA repair taking place at dysfunctional telomeres (Fig. 2). Damaged telomeres are processed by the two pathways that repair most DSBs: nonhomologous end-joining (NHEJ) and homology-directed repair (HDR). Both have potentially detrimental outcomes.

When the shelterin component TRF2 is inhibited or when telomeres become too short, chromosome end fusions are formed. Genetic dissection of the fusions generated by TRF2 loss indicates that they are dependent on DNA ligase IV, implicating the NHEJ pathway (Smogorzewska et al. 2002; Celli and de Lange 2005). The importance of the NHEJ pathway in this context is 
that it generates covalently joined chromosomes that are not readily resolved during mitosis. The resulting problems in anaphase are one of the sources of telomere-related genome instability. A challenge in telomere biology is to understand the mechanism by which shelterin and other telomere-associated factors impede NHEJ and thereby prevent telomere-related genome instability. The most likely explanation is that the t-loop structure itself provides a major hurdle for NHEJ. NHEJ involves the loading of $\mathrm{Ku} 70 / 80$ on a DNA end, which is not available in the t-loop configuration.

HDR at telomeres can also threaten genome integrity. Inappropriate recombination between two telomeres can elongate one telomere at the expense of another. Furthermore, HDR between a telomere and a chromosome-internal stretch of telomere-related sequences can result in translocations, inversions, and deletions (see Fig. 4 below).

\section{Telomere-initiated BFB Cycles Generating Loss of Heterozygosity, Amplification, and Non-reciprocal Translocations}

A main source of telomere-related chromosomal aberrations are the dicentric chromosomes formed when damaged telomeres are processed by NHEJ. Dicentrics are unstable, except when the two centromeres are so close that they function in concert. End-to-end fusions in human cells usually produce fused chromosomes with two independently functioning centromeres. Such dicentrics enter the so-called breakage-fusion-bridge (BFB) cycles originally described by Barbara McClintock (1941). Upon breakage of the dicentric, the newly formed broken ends can initiate a second round of fusion, resulting in another dicentric chromosome and further BFB cycles. Unless the broken ends are healed with a functional telomere, the cells will have ongoing genome instability.

The BFB cycles initiated by a dicentric chromosome can have three outcomes pertinent to cancer genetics: gene losses (LOH), gene amplification, and non-reciprocal translocations (NRTs) (Fig. 3). LOH at cancer-relevant loci can occur upon breakage of a dicentric and concomitant asymmetric segregation of chromosome segments. Gene amplification is also a predicted outcome of BFB cycles, but requires the particularity that the fusion take place after DNA replication and involves sister chromatids (Fig. 3 ). BFB-driven gene amplification generates amplicons organized in inverted repeats, a structure frequently encountered in cancer. The integrity of the genome can further deteriorate when a broken dicentric recombines with another chromosome, giving rise to NRTs.

\section{Inversions, Translocations, and Deletions}

HDR of telomeres can induce a different set of aberrations (Fig. 4). Dysfunctional telomeres can recombine with each other, potentially giving rise to uncontrolled changes in telomere length. Since sequences with substantial homology to telomeric DNA occur at interstitial sites, a dysfunctional telomere could recombine with such an internal stretch of telomeric DNA on the same or
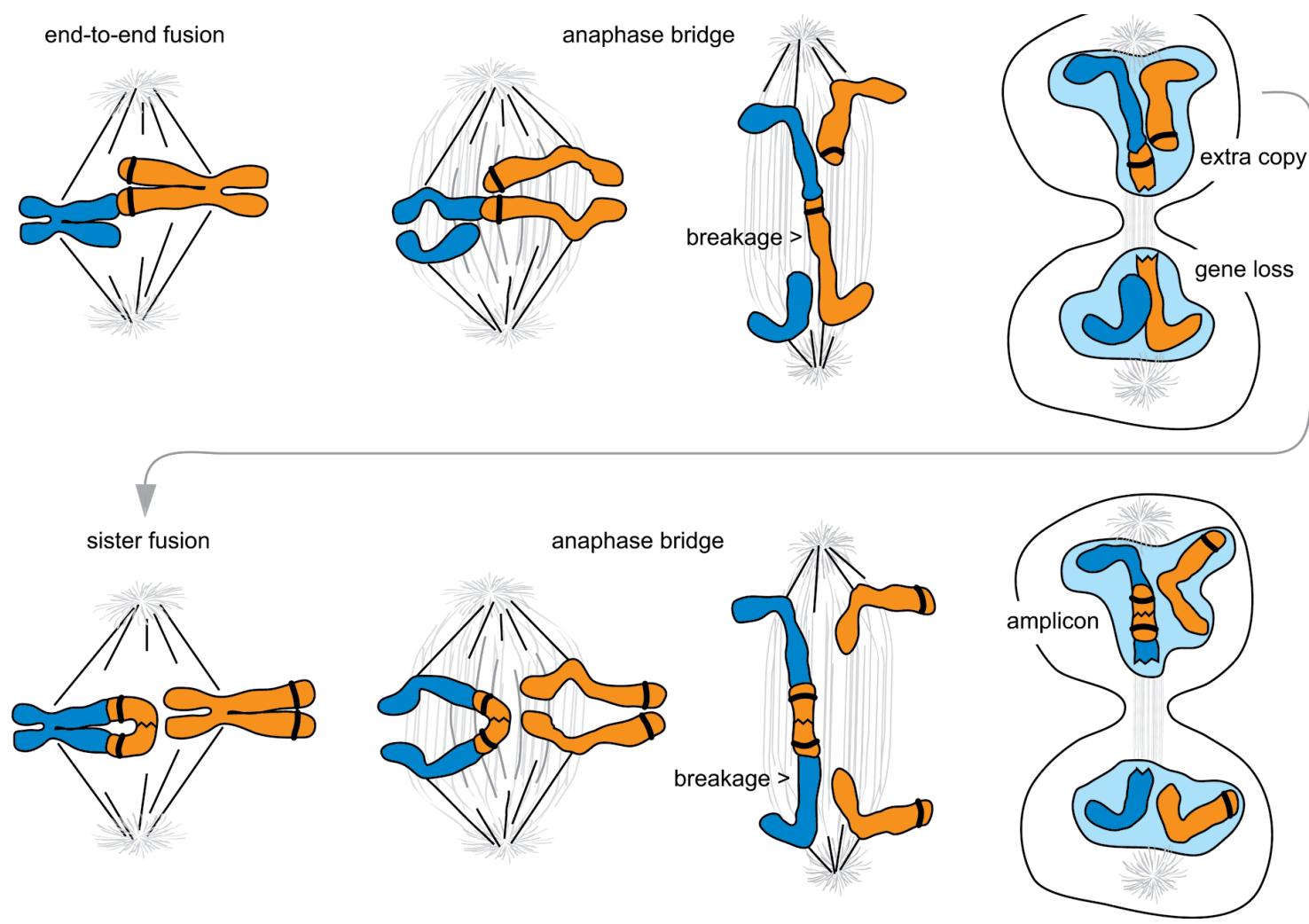

Figure 3. Consequences of telomere fusion and dicentric chromosome formation. 
another chromosome. These events can generate inversions, deletions, and NRTs (Fig. 4). Indirect evidence for the participation of telomeres in HDR events with chromosome internal telomeric DNA has been obtained from $\mathrm{ERCC}^{-/-}$deficient cells (Zhu et al. 2003). These cells form extrachromosomal fragments containing telomeric DNA, called telomeric DNA-containing double-minute chromosomes, TDMs, which are the predicted product of homologous recombination between interstitial telomeric DNA and a dysfunctional telomere (see Fig. 4C).

\section{Telomere-related Tetraploidy and Aneuploidy}

Telomere dysfunction is also a potential source of aneuploidy because damaged telomeres can induce endoreduplication (Fig. 5). The tetraploid cells formed by endoreduplication are the likely precursor to aneuploid genomes (Fig. 1). How cells manage to enter $\mathrm{S}$ phase without completing mitosis is not known. It appears that reentry into $\mathrm{S}$ phase occurs before anaphase. This follows from analysis of primary human cells which have become tetraploid in response to TRF2 inhibition (Smogorzewska and de Lange 2002). The metaphase spreads of such cells often show the presence of diplochromosomes, made up of four chromatids that are closely apposed or connected at the centromere. This indicates that the centromeric cohesin was still present at the time that the cell entered the second round of DNA replication. Since the centromeric cohesin is degraded at anaphase, the presence of diplochromosomes indicates that endoreduplication took place in a cell that had passed through $\mathrm{S}$ phase but had not yet entered anaphase. Tetraploid cells with multiple centrosomes are also observed in primary cells undergoing replicative senescence and appear to be a general outcome of telomere dysfunction. However, the frequency of these events is generally low, affecting at most $15 \%$ of the population.

Once a cell has become tetraploid, chromosome missegregation can generate aneuploid daughter cells (Fig.
1). A tetraploid cell is better able to survive genome damage, since loss of essential functions is less likely. Thus, tetraploidy and its associated aneuploidy form an ideal setting for the accumulation of oncogenic lesions.

\section{Repression of Telomere-related Genome Instability by DNA Damage Checkpoints}

Telomere-related genome instability can be prevented by the activation of the DNA damage response resulting in the culling of cells with dysfunctional telomeres. This telomere damage response has also been invoked as a pathway that limits the proliferative potential of incipient tumor cells once their telomere reserve has been depleted. It is therefore important to understand how cells detect dysfunctional telomeres. Recent data have shown that damaged telomeres activate the canonical DNA damage response (Fig. 2). The ATM kinase is activated, resulting in phosphorylation of Chk2, up-regulation of $\mathrm{p} 53$, and induction of $\mathrm{p} 21$. The DNA damage response can be detected at the dysfunctional telomeres themselves in the form of the so-called Telomere dysfunction Induced Foci (TIFs), which contain DNA damage response markers such as the Mre11 complex, 53BP1, and $\gamma$-H2AX (d'Adda di Fagagna et al. 2003; Takai et al. 2003). Activation of the ATM pathway by damaged telomeres can block entry into $S$ phase through p21-mediated inhibition of Cdk2-cycE, and p53 can induce apoptosis or senescence if the telomere damage persists.

Although the ATM kinase is a prominent transducer of the telomere damage signal, the ATR kinase, and possibly other PIKKs, can respond to dysfunctional telomeres as well (Herbig et al. 2004). For example, A-T cells retain the ability to arrest in response to dysfunctional telo-meres, and global inhibition of PIKKs with caffeine and wortmannin is required to extinguish the TIFs (Takai et al. 2003). Redundancy in the telomere damage signal is also present at the level of the effectors. Most data suggest that
A

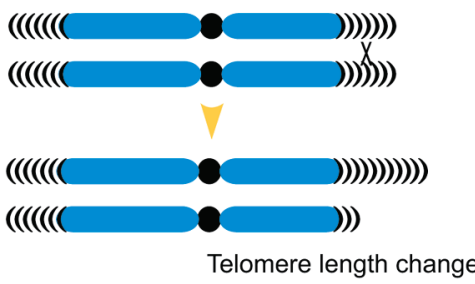

C

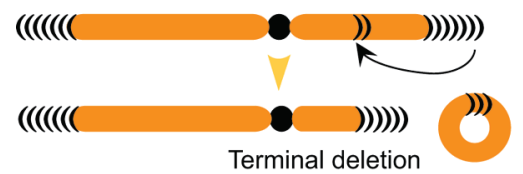

B

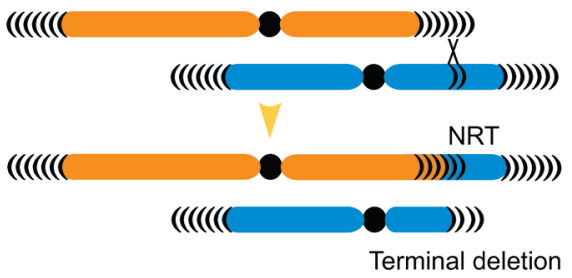

$\mathrm{D}$

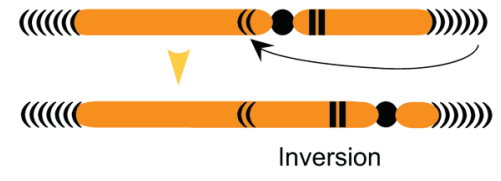

Figure 4. Potential consequences of homology-directed repair at dysfunctional telomeres. $(A)$ Telomere sister chromatid exchanges can elongate one telomere at the expense of another. $(B)$ HDR involving a telomere and interstitial telomeric DNA on another chromosome can give rise to terminal deletions and NRTs. $(C, D)$ Recombination between a telomere and interstitial telomeric DNA on the same chromosome can give rise to a terminal deletion and an acentric fragment or an inversion, depending on the orientation of the interstitial telomeric tract. 


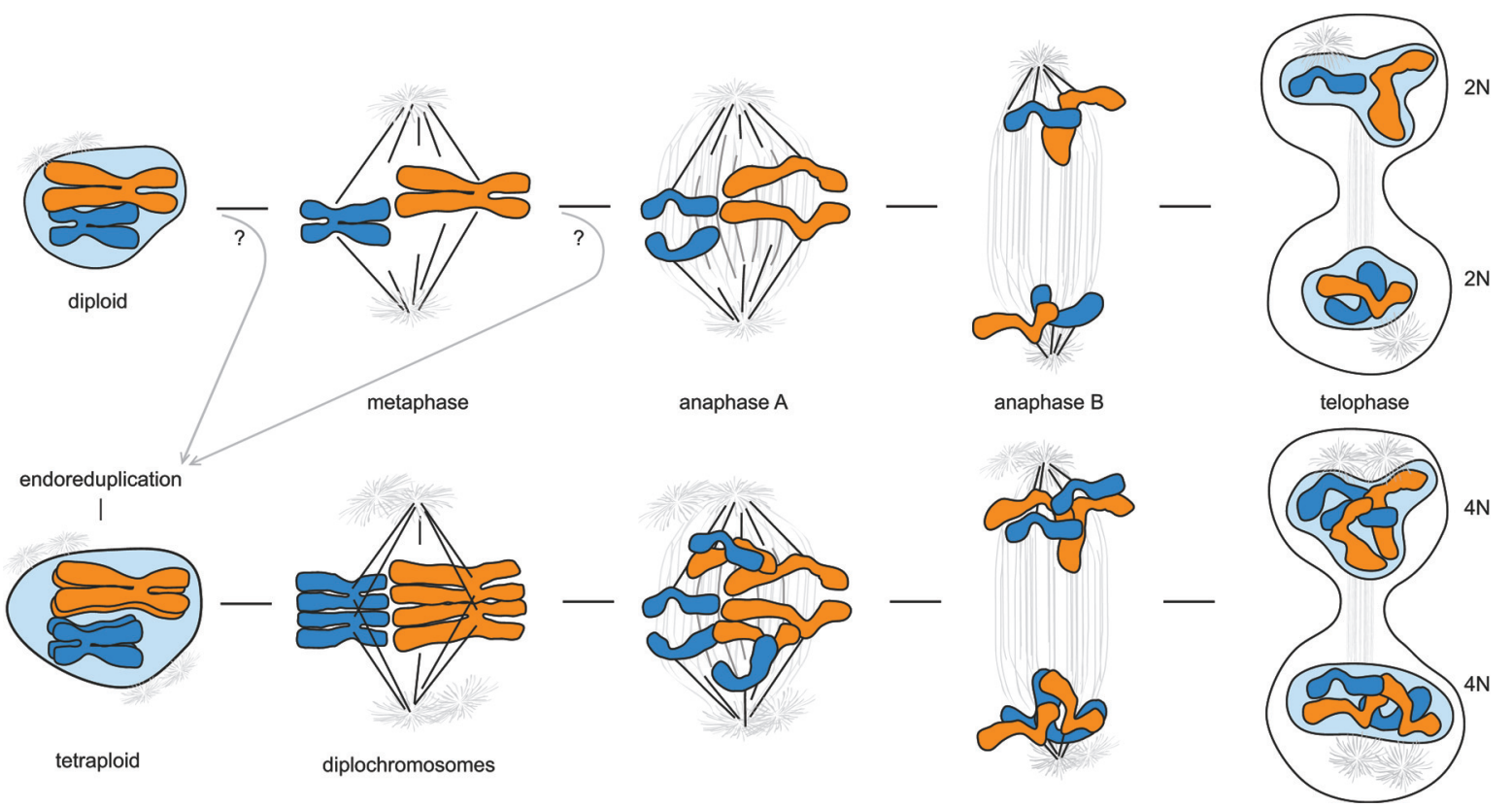

Figure 5. Endoreduplication and formation of tetraploid cells as a consequence of telomere dysfunction.

the p53 pathway is the dominant effector of telomere damage as it is for general DNA damage. However, p53-deficient fibroblasts, although dampened in their response to dysfunctional telomeres, still have the ability to undergo senescence. This secondary pathway is dependent on $\mathrm{p} 16^{\mathrm{INK} 4 \mathrm{a}}$. Ablation of both $\mathrm{p} 53$ and $\mathrm{p} 16$ is required to allow fibroblasts with telomere damage to enter $\mathrm{S}$ phase unimpeded (Jacobs and de Lange 2004; for a contrasting view, see Herbig et al. 2004). The telomere damage response is also abrogated in cells lacking p21, consistent with the proposal that $\mathrm{p} 21$ is the ultimate arbiter of $\mathrm{G}_{1} / \mathrm{S}$ regulation in response to DNA damage (Brown et al. 1997).

\section{Synthesis and Scenario}

What is known about telomere dynamics, telomere function, checkpoint status, and telomerase activity can be synthesized into a scenario that plays out early in tumorigenesis before invasive characteristics have been attained. This scenario describes three distinct stages of telomere dysfunction, each with different consequences for tumorigenesis.

1. Telomere attrition and p53-dependent tumor suppression. Telomere shortening in the early stages of tumorigenesis will eventually induce a DNA damage response, and the accompanying apoptosis or senescence can block tumorigenic potential. In the epithelial compartment and in lymphoid cells, apoptosis is the predominant outcome of telomere dysfunction, whereas senescence is observed in fibroblasts. Although mouse models (see below) suggest a tumor-suppressive effect of shortening telomeres, it is not yet clear whether telomere-driven apoptosis and senescence contribute to tumor suppression in humans. Because both pathways rely heavily on p53 activation, loss of p53 (or other components of this pathway) would curb the immediate tumor-suppressive effect of telomere shortening. In this regard, the order of events is crucial. Is p53 still functional when the telomeres become too short? The answer depends on the replicative history of the cells and the other challenges faced by the transformed cells. For instance, selection for loss of p53 function can occur when hyperplastic and neoplastic growths experience hypoxia (Graeber et al. 1996) or when cells experience a DNA damage response due to inappropriate entry into S phase (Bartkova et al. 2005; Gorgoulis et al. 2005). In p53-deficient fibroblasts, p16 can impede proliferation in response to telomere dysfunction, but it is not yet clear whether this second effector can block other cell types from proliferating beyond the telomere barrier (Jacobs and de Lange 2005). Thus, this first stage in the telomere-cancer scenario is still speculative in human cancer.

2. Crisis due to lethal levels of telomere-related genome instability. Several types of selective pressure, including telomere dysfunction, can explain the emergence of p53-deficient cells in the early stages of tumorigenesis. Such cells are expected to proliferate even if some of their telomeres are defective. If the cell has become tetraploid due to the initial telomere damage response, it may tolerate a considerable level of telomere-related genome instability. Chromosome non-disjunction and ongoing BFB cycles would not necessarily generate daughter cells with lethal genetic deficiencies.

Upon further proliferation, the genome will become increasingly unstable. As more telomeres shorten beyond the minimal functional length, more chromosome end fusions and BFB cycles will follow. The accumulating stress on the genome eventually will curb the proliferative potential of these cells, precipitating a 
growth crisis. There are no clear genetic determinants of the way in which a cell population perishes at this point, suggesting nonspecific cell death events due to different genetic deficiencies in the daughter cells. At this stage, cells can only survive if telomerase heals the dysfunctional telomeres and adds telomeres to the DSBs that have resulted from telomere-related chromosome breakage.

3. Telomerase keeps telomeres on the verge. Telomerase up-regulation, the final step in this scenario, often occurs early in tumor development. For instance, in breast cancer, telomerase activity becomes robust at the DCIS stage, before an invasive phenotype is acquired (Herbert et al. 2001; Meeker and Argani 2004). At this stage, telomere attrition has already removed much of the telomere reserve, providing a selective pressure for a telomere maintenance system. A similar scenario seems to apply to other epithelial cancers (Meeker et al. 2004). Once telomerase is activated, the level of telomere-related genome instability will diminish and BFB cycles can be abrogated by de novo telomere addition. The abrogation of BFB cycles will provide telomerase-positive cells with a considerable proliferative advantage.

Even though telomerase is expressed, tumor telomeres tend to be short (de Lange et al. 1990; Hastie et al. 1990). Work on telomere length maintenance has revealed how cells can attain short but stable telomeres. The length of telomeres is determined by at least three parameters: the activity telomerase, the rate of telomere shortening, and a telomere length homeostasis pathway governed by shelterin. Shelterin is part of a negative feedback loop which inhibits telomerase at a telomere that has become too long (Smogorzewska and de Lange 2004). As more shelterin is loaded onto a telomere, telomerase's ability to act on its end is diminished. The primary role of this homeostasis pathway is thought to control telomere length in the germ line and during early development so that the appropriate telomere length is transferred to all offspring. However, telomere length homeostasis is also operational in human tumor cell lines and presumably also affects telomere length in tumors in vivo. Thus, high levels of shelterin can keep tumor telomeres at a short length setting, even though telomerase is highly active.

The short telomere length of most human tumors suggests that their telomeres never regain full function, and many human tumor cell lines show evidence of partial telomere dysfunction (e.g., telomere fusions). The mild genome instability associated with such partially functional telomeres could confer a selective advantage during tumor progression while not curbing the proliferation rate of the cells.

\section{Inherent in the Scenario: A Transient Burst of Telomere-related Genome Instability}

One of the most compelling arguments in favor of telomere dysfunction as a source of genome instability in cancer is based on its transient occurrence. A mutator phenotype is favored when extrinsic or intrinsic forces require generation of variants. Through hitchhiking with selected mutations, a mutator phenotype can become fixed in the population. Such persistence of a mutator allele comes at a cost, since most mutations are deleterious. A brief episode of high mutation rate followed by return to a more stable genome would avoid a potential mutational load that might hamper proliferation. In this regard, telomere dysfunction is different from other sources of genome instability, since it is reversible through the upregulation of telomerase. Upon acquisition of sufficient telomerase levels, this period of telomere-related scrambling will end, resulting in more stable, yet altered, genomes. The notion that tumors develop through a brief period of telomere dysfunction that generates extensive genetic diversity is borne out by data on genomic alterations during the development of breast cancer (Chin et al. 2004).

\section{Alternative Scenarios: Tumors Lacking Telomere- related Genome Instability}

If telomerase is active before malignant transformation, telomere-related genome instability is less likely to occur. Examples are the lymphomas and leukemias, probably arising in telomerase-competent cell types. The genomes of these types of cancer, while carrying telltale balanced translocations, often lack the complex karyotypes seen in carcinomas (Hilgenfeld et al. 1999; Roschke et al. 2003). Another scenario is represented by the solid tumors of early childhood, which may have ample telomere reserve and emerge as clinically detectable malignancies in a relatively short time period, thus limiting the impact of replicative telomere attrition. For example, retinoblastomas have simple karyotypes and often lack telomerase (Gupta et al. 1996). Neuroblastoma can also arise without telomerase activation and, in this tumor type, absence of telomerase is correlated with better outcome (Hiyama et al. 1995, 1997; Streutker et al. 2001).

\section{Modeling in the Mouse}

In order to mimic the telomere biology of human cells, telomerase-deficient mice have to be propagated over several generations so that their telomeres become sufficiently short. When such mice are challenged with DMBA/TPA to promote skin tumors, short telomeres have a tumor-suppressive effect (Gonzalez-Suarez et al. 2000). Similar results were obtained in the INK4a(delta 2/3) mouse model (Greenberg et al. 1999), several models for hepatocellular carcinoma (Farazi et al. 2003), and ApcMin-induced intestinal carcinoma (Rudolph et al. 2001). In these settings, telomere shortening has little or no effect on the incidence of early-stage lesions; rather, the telomere tumor suppressor pathway appears to limit tumor progression.

Although telomere attrition can limit tumor outgrowth in several mouse models, dysfunctional telomeres promote tumorigenesis in mice with a deficient p53 pathway. 
This difference is likely to be due to the role of p53 in enforcing cell cycle arrest after telomere damage. Mouse cells that lack p53 continue to proliferate despite telomere dysfunction, so that the tumor-suppressive aspect of telomere dysfunction is abrogated. In that setting, the ability of telomere dysfunction to promote tumorigenesis emerges (Chin et al. 1999). A seminal experiment showed that dysfunctional telomeres specifically promote malignant transformation of epithelial cells (Artandi et al. 2000). The telomere attrition generated in the TTERC $^{-/-}$ mouse induced a remarkable shift in the tumor spectrum associated with heterozygosity for $\mathrm{p} 53$. Whereas $\mathrm{p} 53^{+/-}$ mice which usually develop lymphomas and sarcomas, when combined with telomere dysfunction, $\mathrm{p} 53^{+/-}$status leads to a predominance of carcinomas. As expected, these tumors have lost the wild-type p53 allele. Karyotypic analysis indicates a higher burden of genome rearrangements in the tumors, including both clonal and nonclonal NRTs. Furthermore, these tumors show amplification and $\mathrm{LOH}$, as predicted based on the known outcomes of telomere dysfunction.

Although the telomerase knockout mouse model has been extremely informative, there are two aspects of human tumorigenesis it does not reflect. In this model, tumorigenesis takes place in the context of persistent telomere dysfunction. Telomerase is absent and cannot be activated. As argued above, human tumorigenesis is more likely to progress through a transient burst of telomererelated genome instability, followed by telomerase-mediated (partial) stabilization of the genome. A second potential difference is found in the telomere damage signaling pathway in murine and human cells. Human fibroblasts can respond to telomere damage through the up-regulation of either $\mathrm{p} 53$ or $\mathrm{p} 16$, whereas mouse fibroblasts lack the p16 response. Therefore, loss of p53 is sufficient to abrogate the cell cycle arrest upon telomere damage in the mouse system. The challenge will be to create mouse models that address these issues and more accurately reflect telomere biology in human cells.

\section{ACKNOWLEDGMENTS}

The research in my laboratory is supported by grants from the National Institutes of Health (CA76027, AG16642, and GM49046) and by a grant from the Breast Cancer Research Foundation.

\section{REFERENCES}

Artandi S.E., Chang S., Lee S.L., Alson S., Gottlieb G.J., Chin L., and DePinho R.A. 2000. Telomere dysfunction promotes non-reciprocal translocations and epithelial cancers in mice. Nature 406: 641.

Bartkova J., Horejsi Z., Koed K., Kramer A., Tort F., Zieger K., Guldberg P., Sehested M., Nesland J.M., Lukas C., Orntoft T., Lukas J., and Bartek J. 2005. DNA damage response as a candidate anti-cancer barrier in early human tumorigenesis. $\mathrm{Na}$ ture 434: 864.

Brown J.P., Wei W., and Sedivy J.M. 1997. Bypass of senescence after disruption of p21CIP1/WAF1 gene in normal diploid human fibroblasts. Science 277: 831.

Celli G. and de Lange T. 2005. DNA processing not required for
ATM-mediated telomere damage response after TRF2 deletion. Nat. Cell Biol. 7: 712.

Chen J.-L. and Greider C.W. 2005. Telomerase biochemistry and biogenesis. In Telomeres, 2nd edition (ed. T. de Lange et al.), p. 49. Cold Spring Harbor Laboratory Press, Cold Spring Harbor, New York.

Chin K., De Solorzano C.O., Knowles D., Jones A., Chou W., Rodriguez E.G., Kuo W.L., Ljung B.M., Chew K., Myambo K., Miranda M., Krig S., Garbe J., Stampfer M., Yaswen P., Gray J.W., and Lockett S.J. 2004. In situ analyses of genome instability in breast cancer. Nat. Genet. 36: 984.

Chin L., Artandi S.E., Shen Q., Tam A., Lee S.L., Gottlieb G.J., Greider C.W., and DePinho R.A. 1999. p53 deficiency rescues the adverse effects of telomere loss and cooperates with telomere dysfunction to accelerate carcinogenesis. Cell 97: 527.

Cristofari G. and Lingner J. 2005. The telomerase ribonucleoprotein particle. In Telomeres, 2 nd edition (ed. T. de Lange et al.), p. 21. Cold Spring Harbor Laboratory Press, Cold Spring Harbor, New York.

d'Adda di Fagagna F., Reaper P.M., Clay-Farrace L., Fiegler H., Carr P., Von Zglinicki T., Saretzki G., Carter N.P., and Jackson S.P. 2003. A DNA damage checkpoint response in telomere-initiated senescence. Nature 426: 194.

de Lange T. 1995. Telomere dynamics and genome instability in human cancer. In Telomeres (ed. E.H. Blackburn and C.W. Greider), p. 265. Cold Spring Harbor Laboratory Press, Cold Spring Harbor, New York.

. 2005a. Mammalian telomeres. In Telomeres, 2nd edition (ed. T. de Lange et al.), p. 387. Cold Spring Harbor Laboratory Press, Cold Spring Harbor, New York.

2005b. Shelterin: The protein complex that shapes and safeguards human telomeres. Genes Dev. 19: 2100.

de Lange T., Shiue L., Myers R.M., Cox D.R., Naylor S.L., Killery A.M., and Varmus H.E. 1990. Structure and variability of human chromosome ends. Mol. Cell. Biol. 10: 518.

Farazi P.A., Glickman J., Jiang S., Yu A., Rudolph K.L., and DePinho R.A. 2003. Differential impact of telomere dysfunction on initiation and progression of hepatocellular carcinoma. Cancer Res. 63: 5021.

Gonzalez-Suarez E., Samper E., Flores J.M., and Blasco M.A. 2000. Telomerase-deficient mice with short telomeres are resistant to skin tumorigenesis. Nat. Genet. 26: 114.

Gorgoulis V.G., Vassiliou L.V., Karakaidos P., Zacharatos P., Kotsinas A., Liloglou T., Venere M., Ditullio R.A.J., Kastrinakis N.G., Levy B., Kletsas D., Yoneta A., Herlyn M., Kittas C., and Halazonetis T.D. 2005. Activation of the DNA damage checkpoint and genomic instability in human precancerous lesions. Nature 434: 907.

Graeber T.G., Osmanian C., Jacks T., Housman D.E., Koch C.J., Lowe S.W., and Giaccia A.J. 1996. Hypoxia-mediated selection of cells with diminished apoptotic potential in solid tumours. Nature 379: 88.

Greenberg R.A., Chin L., Femino A., Lee K.H., Gottlieb G.J., Singer R.H., Greider C.W., and DePinho R.A. 1999. Short dysfunctional telomeres impair tumorigenesis in the INK4a(delta2/3) cancer-prone mouse. Cell 97: 515.

Gupta J., Han L.P., Wang P., Gallie B.L., and Bacchetti S. 1996. Development of retinoblastoma in the absence of telomerase activity. J. Natl. Cancer Inst. 88: 1152.

Hastie N.D., Dempster M., Dunlop M.G., Thompson A.M., Green D.K., and Allshire R.C. 1990. Telomere reduction in human colorectal carcinoma and with ageing. Nature 346: 866.

Herbert B.S., Wright W.E., and Shay J.W. 2001. Telomerase and breast cancer. Breast Cancer Res. 3: 146.

Herbig U., Jobling W.A., Chen B.P., Chen D.J., and Sedivy J.M. 2004. Telomere shortening triggers senescence of human cells through a pathway involving ATM, p53, and p21(CIP1), but not p16(INK4a). Mol. Cell 14: 501.

Hilgenfeld E., Padilla-Nash H., Schrock E., and Ried T. 1999. Analysis of B-cell neoplasias by spectral karyotyping (SKY). Curr. Top. Microbiol. Immunol. 246: 169.

Hiyama E., Hiyama K., Yokoyama T., Matsuura Y., Piatyszek M.A., and Shay J.W. 1995. Correlating telomerase activity lev- 
els with human neuroblastoma outcomes. Nat. Med. 1: 249.

Hiyama E., Hiyama K., Ohtsu K., Yamaoka H., Ichikawa T., Shay J.W., and Yokoyama T. 1997. Telomerase activity in neuroblastoma: Is it a prognostic indicator of clinical behaviour? Eur. J. Cancer 33: 1932.

Jacobs J.J. and de Lange T. 2004. Significant role for p16(INK4a) in p53-independent telomere-directed senescence. Curr. Biol. 14: 2302

2005. p16INK4a as a second effector of the telomere damage pathway. Cell Cycle 4: 1360.

Lucito R., Healy J., Alexander J., Reiner A., Esposito D., Chi M., Rodgers L., Brady A., Sebat J., Troge J., West J.A., Rostan S., Nguyen K.C., Powers S., Ye K.Q., Olshen A., Venkatraman E., Norton L., and Wigler M. 2003. Representational oligonucleotide microarray analysis: A high-resolution method to detect genome copy number variation. Genome Res. 13: 2291.

McClintock B. 1941. The stability of broken ends of chromosomes in Zea mays. Genetics 26: 234.

Meeker A.K. and Argani P. 2004. Telomere shortening occurs early during breast tumorigenesis: A cause of chromosome destabilization underlying malignant transformation? J. Mammary Gland Biol. Neoplasia 9: 285.

Meeker A.K., Hicks J.L., Iacobuzio-Donahue C.A., Montgomery E.A., Westra W.H., Chan T.Y., Ronnett B.M., and De Marzo A.M. 2004. Telomere length abnormalities occur early in the initiation of epithelial carcinogenesis. Clin. Cancer Res. 10: 3317.

Neumann A.A. and Reddel R.R. 2005. Telomerase-independent maintenance of mammalian telomeres. In Telomeres (ed. T. de Lange et al.), p. 163. Cold Spring Harbor Laboratory Press, Cold Spring Harbor, New York.

Pinkel D., Segraves R., Sudar D., Clark S., Poole I., Kowbel D., Collins C., Kuo W.L., Chen C., Zhai Y., Dairkee S.H., Ljung
B.M., Gray J.W., and Albertson D.G. 1998. High resolution analysis of DNA copy number variation using comparative genomic hybridization to microarrays. Nat. Genet. 20: 207.

Roschke A.V., Tonon G., Gehlhaus K.S., McTyre N., Bussey K.J., Lababidi S., Scudiero D.A., Weinstein J.N., and Kirsch I.R. 2003. Karyotypic complexity of the NCI-60 drug-screening panel. Cancer Res. 63: 8634.

Rudolph K.L., Millard M., Bosenberg M.W., and DePinho R.A. 2001. Telomere dysfunction and evolution of intestinal carcinoma in mice and humans. Nat. Genet. 28: 155.

Shay J.W. and Wright W.E. 2005. Telomerase and human cancer. In Telomeres, 2 nd edition (ed. T. de Lange et al.), p. 81. Cold Spring Harbor Laboratory Press, Cold Spring Harbor, New York.

Smogorzewska A. and de Lange T. 2002. Different telomere damage signaling pathways in human and mouse cells. EMBO J. 21: 4338 .

2004. Regulation of telomerase by telomeric proteins. Annu. Rev. Biochem. 73: 177.

Smogorzewska A., Karlseder J., Holtgreve-Grez H., Jauch A., and de Lange T. 2002. DNA ligase IV-dependent NHEJ of deprotected mammalian telomeres in G1 and G2. Curr. Biol. 12: 1635.

Streutker C.J., Thorner P., Fabricius N., Weitzman S., and Zielenska M. 2001. Telomerase activity as a prognostic factor in neuroblastomas. Pediatr. Dev. Pathol. 4: 62.

Takai H., Smogorzewska A., and de Lange T. 2003. DNA damage foci at dysfunctional telomeres. Curr. Biol. 13: 1549.

Zhu X.D., Niedernhofer L., Kuster B., Mann M., Hoeijmakers J.H., and de Lange T. 2003. ERCC1/XPF removes the 3' overhang from uncapped telomeres and represses formation of telomeric DNA-containing double minute chromosomes. Mol. Cell. 12: 1489. 


\section{$8_{8}^{\infty} \mathrm{CSH} \&$ Cold Spring Harbor Symposia SYMPOSIA on Quantitative Biology}

\section{Telomere-related Genome Instability in Cancer}

\section{T. DE LANGE}

Cold Spring Harb Symp Quant Biol 2005 70: 197-204

Access the most recent version at doi:10.1101/sqb.2005.70.032

References This article cites 36 articles, 9 of which can be accessed free at: http://symposium.cshlp.org/content/70/197.full.html\#ref-list-1

\section{License}

Email Alerting Receive free email alerts when new articles cite this article - sign up in Service the box at the top right corner of the article or click here. 